\title{
Critical Care Ultrasonography: essential skill for a bedside physician.
}

\section{Gentle S Shrestha}

Critical care ultrasonography (CCUS) is a bedside technique performed by the clinician at point of care. Conceptually, CCUS functions as an extension of traditional physical examination by allowing the intensivist to visualize the anatomy and function of various organ systems in real time. It overcomes the limitations of ultrasonography performed by radiology or cardiology services on consultative basis. The inevitable delay in obtaining the study is avoided and the dissociation between the individual interpreting the study and the physician treating the patient at bedside is eliminated. The beauty of CCUS lies in that, the focused examinations can be repeated as required at bedside, without the need to transfer to radiology suite and without additional exposure to radiation. The repeat examination can track effects of therapy and evolution of disease with time. ${ }^{1}$

CCUS is evolving from an optional to an indispensable tool for managing critically ill patients. General CCUS incorporates competence in pleural, lung, abdominal and vascular ultrasonography. Basic critical care echocardiography incorporates qualitative and target oriented ultrasonographic cardiac evaulation at bedside. ${ }^{2}$ It is suggested that general CCUS and basic critical care echocardiography should be mandatory in the curriculum of ICU physicians. Critical Care Societies should support the implementation of training in CCUS in its own country ${ }^{3}$ and should work to achieve national consensus on standards of training, quality assurance and maintenance of competence in CCUS for intensivists and critical care trainees. ${ }^{4}$

CCUS can function as a valuable adjunct to physical examination for evaluation of critically ill patient with undifferentiated shock, which can be one of the most challenging issues in daily practice. ${ }^{5}$ In ICU patients, bedside lung ultrasound has a considerably better diagnostic performance than chest X-ray for the diagnosis of common pathological conditions and may serve as an alternative to thoracic CT. ${ }^{7}$ Ultrasonography of optic nerve sheath diameter has a good diagnositc accuracy for detecting intracranial hypertension. ${ }^{8}$

Beyond the convincing diagnositc yield of CCUS, it can be a useful bedside tool to guide treatment and to follow the response of therapy. Ultrasonographic measurement of respiratory variation of inferior vena cava diameter is useful in predicting fluid responsiveness in ICU patients. ${ }^{9}$ Bedside lung ultrasound can be a tool to assess the response to antibiotic therapy in patients with ventilator-associated pneumonia. ${ }^{10}$ Diaphragm dysfunction, assessed by bedside ultrasonography has been shown to predict patients with weaning difficulty and weaning failure. ${ }^{11}$

Application of CCUS can enhance safety of common bedside procedures like central venous cannulation, ${ }^{12}$ arterial catheterization $^{13}$ and thoracocentesis. ${ }^{14}$ CCUS can have impact on clinical decision making and therapeutic management. ${ }^{15,16,17}$

CCUS is a teachable and readily learnable skill. Simulation and web-based technologies, when available, can be used for standardization of both ultrasound skills training and competency assessment. ${ }^{18}$

CCUS can be valuable in resource-limited settings where the diagnostic imaging modalities are often constrained and frequently limited to plain film radiography. As ultrasound machines become more portable and affordable, and with the advent of low-cost telemetry, CCUS may have a transformative effect on critical care in resource-limited settings. ${ }^{19}$

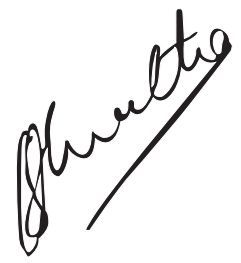

Dr. Gentle Sunder Shrestha

MD, FACC, EDIC, FCCP

Intensivist \& Anaesthesiologist

Department of Anaesthesiology

Tribhuvan University Teaching Hospital

Maharajgunj, Kathmandu, Nepal

gentlesunder@hotmail.com

\section{References:}

1. Cardenas-Garcia J, Mayo PH. Bedside ultrasonography for the intensivist. Crit Care Clin 2015;31:43-66.

2. Mayo PH, Beaulieu Y, Doelken P, Feller-Kopman D, Harrod C, Kaplan A, et al. American College of Chest Physicians/La Société de Réanimation de Langue Française statement on competence in critical care ultrasonography. Chest 2009;135:1050-60.

3. Cholley BP, Mayo PH, Poelaert J, Vieillard-Baron A, Vignon P, Alhamid $\mathrm{S}$, et al. International expert statement on training standards for critical care ultrasonography. Intensive Care Med 2011;37:1077-83.

4. Arntfield R, Millington S, Ainsworth C, Arora R, Boyd J, Finlayson $\mathrm{G}$, et al. Canadian recommendations for critical care ultrasound training and competency. Can Respir J 2014;21:341-5.

5. Perera P, Mailhot T, Riley D, Mandavia D. The RUSH exam: Rapid 
ultrasound in shock in the evaluation of the critically ill. Emerg Med Clin North Am 2010;28:29-56.

6. Lichtenstein DA, Meziere GA. Relevance of lung ultrasound in the diagnosis of acute respiratory failure: the BLUE protocol. Chest 2008; 134:117-25.

7. Xirouchaki N, Magkanas E, Vaporidi K, Kondilli E, Plataki M, Patrianakos A, et al. Lung ultrasound in critically ill patients: comparison with bedside chest readiography. Intensive Care Med 2011;37:1488-93.

8. Dubourg J, Javouhey E, Geeraerts T, Messerer M, Kassai B. Ultrasonography of optic nerve sheath diameter for detection of raised intracranial pressure: a systematic review and meta-analysis. Intensive Care Med 2011;37:1059-68.

9. Zhang Z, Xu X, Ye S, Xu L. Ultrasonographic measurement of the respiratory variation in the inferior vena cava diameter is predictive of fluid responsiveness in critically ill patients: systematic review and meta-analysis. Ultrasound Med Biol 2014;40:845-53.

10. Bouhemad B, Liu ZH, Arbelot C, Zhang M, Ferrari F, Le-Guen M, et al. Ultrasound assessment of antibiotic-induced pulmonary reaeration in ventilator-associated pneumonia. Crit Care Med 2010;38:84-92.

11. Kim WY, Suh HJ, Hong SB, Koh Y, Lim CM. Diaphragm dysfunction assessed by ultrasonography: influence on weaning from mechanical ventilation. Crit Care Med 2011;39:2627-30.

12. Brass P, Hellmich M, Kolodziej L, Schick G, Smith AF. Ultrasound guidance versus anatomical landmarks for internal jugular vein catheterization. Cochrane Database Syst Rev 2015;1:CD006962.
13. Zochios VA, Wilkinson J, Dasgupta K. The role of ultrasound as an adjunct to arterial catheterization in critically ill surgical and intensive care unit patients. J Vasc Access 2014;15:1-4.

14. Gordon CE, Feller-Kopman D, Balk EM, Smetana GW. Pneumothorax following thoracocentesis: a systematic review and meta-analysis. Arch Intern Med 2010;170:332-9.

15. Xirouchaki N, Kondili E, Prinianakis G, Malliotakis P, Georgopoulos D. Impact of lung ultrasound on clinical decision making in critically ill patients. Intensive Care Med 2014;40:57-65.

16. Haydar SA, Moore ET, Higgins GL $3^{\text {rd }}$, Irish CB, Owens WB, Strout TD. Effect of bedside ultrasonography on the certainty of physician clinical decisionmaking for septic patients in the emergency department. Ann Emerg Med 2012;60:346-58.

17. Shrestha GS, Khanal A, Paudel S. Avoiding iatrogenic harm by integrating physical examination findings with a point-of-care lung ultrasonography. Indian J Crit Care Med 2015;19:243-4.

18. Lewiss RE, Hoffmann B, Beaulieu Y, Phelan MB. Point-of-care ultrasound education: the increasing role of simulation and multimedia resources. J Ultrasound Med 2014;33:27-32.

19. Henwood PC, Mackenzie DC, Rempell JS, Murray AF, Leo MM, Dean AJ, et al. A practical guide to self-sustaining point-of-care ultrasound education programs in resource-limited settings. Ann Emerg Med 2014;64:277-85. 